\title{
Administration of live, but not inactivated, Faecalibacterium prausnitzii has a preventive effect on dextran sodium sulfate-induced colitis in mice
}

\author{
YUJIRO KAWADE ${ }^{1}$, MISAKI SAKAI ${ }^{1}$, MARIKO OKAMORI ${ }^{1}$, MAYUKO MORITA ${ }^{2,3}$, KATSURA MIZUSHIMA ${ }^{2}$, \\ TOMOHIRO UEDA ${ }^{2}$, TOMOHISA TAKAGI ${ }^{2}$, YUJI NAITO ${ }^{2}$, YOSHITO ITOH ${ }^{2,3}$ and TAKASHI SHIMADA ${ }^{1,3}$ \\ ${ }^{1}$ Central Research Laboratories, Nichinichi Pharmaceutical Co., Ltd., Iga, Mie 518-1417; \\ Departments of ${ }^{2}$ Molecular Gastroenterology and Hepatology, and ${ }^{3}$ Gastrointestinal Immunology, \\ Kyoto Prefectural University of Medicine, Kamigyo-ku, Kyoto 602-8566, Japan
}

Received December 6, 2018; Accepted April 18, 2019

DOI: $10.3892 / \mathrm{mmr} .2019 .10234$

\begin{abstract}
Faecalibacterium prausnitzii is one of the most abundant bacteria in the human gut microbiota. This bacterium is reported to serve an important role in inflammatory bowel diseases. In the present study, the preventive effects of $F$. prausnitzii on a dextran sodium sulfate (DSS)-induced colitis model in mice were investigated. BALB/c mice were fed with 5\% DSS in drinking water. Administration of live or inactivated $F$. prausnitzii was initiated 7 days prior to the start of DSS feeding. Mucosal cytokines were analyzed by reverse transcription-quantitative PCR. Histological analysis of colon mucosa was also performed. The symptoms of DSS-induced colitis (weight loss, diarrhea, bloody stools and colon shortening) were significantly improved in the group administered live $F$. prausnitzii, but not in the other groups. There were no significant differences in the expression of proinflammatory cytokines; however, the expression of mucosal cytokines appeared to be markedly reduced in the live $F$. prausnitzii-administered group compared with the DSS-fed control. The results suggested that preventive administration of 'live', but not inactivated, F. prausnitzii protected the colon against DSS-induced colitis. Live $F$. prausnitzii were also administered therapeutically following the induction of colitis, resulting in an improved histological score in mice.
\end{abstract}

\section{Introduction}

Faecalibacterium prausnitzii was first isolated from human feces in the early 20th century, and classified under the genus

Correspondence to: Dr Takashi Shimada, Central Research Laboratories, Nichinichi Pharmaceutical Co., Ltd., 239-1 Tominaga, Iga, Mie 518-1417, Japan

E-mail: lab@nichinichi-phar.co.jp

Key words: Faecalibacterium prausnitzii, dextran sulfate sodium, experimental colitis, probiotics, murine colitis model
Fusobacterium (1); however, it was re-classified under the genus Faecalibacterium by Duncan et al (2) in 2002, due to significant differences in the GC content, 16S ribosomal rDNA sequence and oxygen tolerance between these two genera. $F$. prausnitzii is a butyric acid-producing, obligate anaerobic bacillus, and is classified into two molecular phylogroups similar to strains ATCC27768 and A2-165, based on the 16S rDNA sequence (3). F. prausnitzii is reportedly abundant; $F$. prausnitzii comprises $6-8 \%$ of the gut microbiome in healthy humans, but up to $20 \%$ in certain individuals (4-12). Conversely, this bacterium is not detected in early infants (aged 0-6 months); it is first detected at 7 months of age or older (13-16). Recently, F . prausnitzii was reported to serve an important role in inflammatory bowel disease (IBD), including ulcerative colitis (UC) and Crohn's disease (17). Disorders of the gut microbiome serve an important role in IBD $(18,19)$. Numerous studies have reported that the population of $F$. prausnitzii is decreased in the mucosa and feces of patients with IBD, albeit with certain exceptions (18-30). A number of chemically-induced animal models of experimental colitis have been employed to investigate the pathophysiology of IBD (18,31-33). The dextran sodium sulfate (DSS)-induced colitis model is one of the most widely used models, due to the reproducibility of results (31-33). DSS-induced colitis is morphologically characterized by damage to epithelial cells, ulceration, submucosal edema, and the infiltration of granulocytes and mononuclear immune cells (34). Therefore, the DSS-induced colitis model is considered to exhibit features similar to those of human UC. The present study aimed to investigate the preventive and therapeutic potential of $F$. prausnitzii administration.

\section{Materials and methods}

Unless otherwise specified, Reagents were purchased from FUJIFILM Wako Pure Chemical Corporation.

Bacterium. F. prausnitzii ATCC27768 (purchased from ATCC) was grown under anaerobic conditions with $\mathrm{N}_{2}$ gas using the Hungate method (35). The culture medium contained 
the following contents per liter: $10 \mathrm{~g}$ Bacto peptone, $10 \mathrm{~g}$ LAB-LEMCO powder, $3 \mathrm{~g}$ Bacto yeast extract, $5 \mathrm{~g}$ D-glucose, $2.5 \mathrm{~g}$ maltose, $5 \mathrm{~g} \mathrm{NaCl}, 1 \mathrm{~g}$ soluble starch, $1 \mathrm{~g}$ L-cysteine $\mathrm{HCl}, 3 \mathrm{~g}$ sodium acetate and $1 \mathrm{mg}$ resazurin. F. prausnitzii was cultured at $37^{\circ} \mathrm{C}$ until the optical density at $650 \mathrm{~nm}$ reached $>1.5$. Prior to administration to mice, bacterial culture fluid was collected anaerobically with a $1 \mathrm{ml}$ syringe, and was stored at $4^{\circ} \mathrm{C}$ until administration. Inactivation of the bacterium was performed by air mixing. Culture fluid $(30 \mathrm{ml})$ was transferred from the Hungate tube to a $50-\mathrm{ml}$ centrifugation tube, gently mixed well without using a vortex mixer and exposed to air for at least $30 \mathrm{~min}$. Following air mixing, one tube was used as inactivated $F$. prausnitzii (iFP), and another was divided into bacterial cells (FP cells) and supernatant (FP sup.).

Experimental animals and colitis induction. All the experimental procedures using mice were conducted in accordance with the National Institutes of Health guidelines for the use of experimental animals (36). Experiments were performed according to the guidelines for the care and use of laboratory animals approved by Nichinichi Pharmaceutical Co., Ltd. All experimental programs were approved by the Animal Care Committee of Kyoto Prefectural University of Medicine (Kyoto, Japan). BALB/cCrSlc mice (5-week-old males) were purchased from Japan SLC, Inc. A total of 100 mice were used in this study. They were acclimatized for 1 week prior to experiments, and were housed individually in a room maintained at $22^{\circ} \mathrm{C}$ and normal humidity, under a $12: 12$-h day/night cycle throughout the experiments. The mice were allowed free access to rodent chow (CE-2; CLEA Japan, Inc.) and filtered tap water. Experimental colitis was initiated by oral administration of 5\% DSS (molecular weight, 5,000 Da) mixed with tap water. Body weight was measured daily throughout every experiment. The mean body weight was $21.1 \pm 0.8 \mathrm{~g}$ following acclimatization. Health and behavior were monitored daily by measuring body weight. Mice were euthanized within $1 \mathrm{~h}$ if the body weight decreased to $<80 \%$ of the weight at the start of experiments. No animals succumbed prior to meeting the criteria for sacrifice. No other measures were implemented due to the minor distress that may be induced. Research staff were trained in animal care and handling by an animal experiment specialist.

Preventive experiment. A total of two preventive experiments were performed. In the first experiment, mice were divided into 4 groups: Control mice (Cont. group, $\mathrm{n}=9$ ); $F$. prausnitziitreated mice (FP group, n=8); 5\% DSS-treated mice (DSS + Cont. group, $\mathrm{n}=19$ ); and 5\% DSS plus $F$. prausnitzii-treated mice (DSS + FP group, $\mathrm{n}=20$ ). To determine the experimental preventive effects of $F$. prausnitzii on colitis, $0.2 \mathrm{ml} /$ mouse/day $F$. prausnitzii culture fluid containing $10^{8}$ colony forming units (CFU) of live $F$. prausnitzii was administered orally for 7 days, and then DSS was administered for 8 days. F. prausnitzii was not administered during the DSS treatment period. The mice were sacrificed at day 8 by cervical dislocation to minimize suffering. The large intestine of each mouse was removed, and the length from under the cecum to the anus was measured as the colon length. H\&E was used to stain colonic mucosa from mice in the DSS + Cont. $(\mathrm{n}=2)$ and DSS + FP groups $(\mathrm{n}=2)$. Staining was $0.2 \%$ hematoxylin solution for $5 \mathrm{~min}$, rinsing in tap water then $1 \%$ acid alcohol, rinsing in tap water again, rinsing in ethanol, staining for 5 min with $1 \%$ eosin solution, and finally rinsing in ethanol. This was all performed at room temperature Reverse transcription-quantitative PCR (RT-qPCR) analysis was performed on 5 samples/group.

In the second experiment, the effects of inactivated culture fluid, bacterial cells and supernatant were determined using the same methods. Mice were divided into 5 groups: Control mice (Cont. group, $\mathrm{n}=5$ ); 5\% DSS-treated mice (DSS + cont. group, n=4); 5\% DSS plus inactivated $F$. prausnitzii-treated mice (DSS + iFP group, $\mathrm{n}=4$ ); $5 \%$ DSS plus $F$. prausnitzii cell-treated mice (DSS + FP cell group, $\mathrm{n}=5$ ); and 5\% DSS plus F. prausnitzii supernatant (DSS + FP sup. group, $\mathrm{n}=5$ ). Group details are presented in Table I. In the preventive experiment, as one animal in the DSS + Cont. group reached the endpoint criteria, it was sacrificed and its data were excluded; the weight loss was hypothesized to be a result of weakness due to DSS-induced colitis.

Therapeutic experiment. To investigate the therapeutic effects of $F$. prausnitzii on colitis, mice were administered 5\% DSS water for 8 days following the acclimation period to induce DSS-colitis. Administration of 5\% DSS water was then terminated for a recovery period of 3 days. Then, $0.2 \mathrm{ml} / \mathrm{mouse} /$ day of $F$. prausnitzii culture fluid for the DSS + FP group ( $\mathrm{n}=10)$, or fresh culture medium for the DSS + Cont. group $(n=10)$ was administered orally for 7 days. The mice were sacrificed on day 19 by cervical dislocation to minimize suffering. Histological analyses using H\&E staining as above was performed on 5 samples/group. RT-qPCR analysis was performed on 5 samples/group.

Assessment of inflammation in DSS-induced colitis. Mucosal inflammation was assessed using the disease activity index (DAI) score, as illustrated in Table II. The DAI scores were calculated by the sum of the fecal properties and hematochezia scores. The DAI score was measured daily.

Histological analysis. For histological analysis, the colon was fixed in $10 \%$ buffered formalin at room temperature for $\sim 1$ week and embedded in paraffin. Sections (4- $\mu$ m thickness) were prepared and stained with hematoxylin and eosin as above. The severity of histological damage was scored in a blinded manner using a previously published grading system $(37,38)$. Briefly, the histological scores indicated the presence and extent of inflammation and epithelial damage in colitis, as determined according to the system presented in Table III. The total score indicated the sum of the epithelium (E) and infiltration (I) scores, and thus ranged between 0 and 8 (total score $=\mathrm{E}+\mathrm{I})$.

Reverse transcription-quantitative PCR (RT-qPCR). Total RNA was extracted from the mucosa of the large intestine using the acid guanidinium phenol chloroform method with Isogen (Nippon Gene Co., Ltd.) and then reverse transcribed with PrimeScript ${ }^{\mathrm{TM}}$ RT-PCR kit (Takara Bio, Inc.). The reverse transcription conditions were: $65^{\circ} \mathrm{C}$ for $5 \mathrm{~min}$ (annealing step), $42^{\circ} \mathrm{C}$ for $30 \mathrm{~min}$ (reverse transcription step), and then $95^{\circ} \mathrm{C}$ for 5 min (inactivation step). cDNA was subjected to qPCR on the 7300 Real-Time PCR System (Applied Biosystems; Thermo 
Table I. Groups for the preventive experiment.

\begin{tabular}{lll}
\hline Group name & Preventive administration & Drinking water \\
\hline Cont. & Anaerobic medium & Tap water \\
FP & Culture fluid (living) & Tap water \\
DSS + Cont. & Anaerobic medium & DSS water \\
DSS + FP & Culture fluid (living) & DSS water \\
DSS + iFP & Culture fluid (inactivated) & DSS water \\
DSS + FP cell & Bacterial cells (inactivated) & DSS water \\
DSS + FP sup. & Supernatant & DSS water
\end{tabular}

The concentration of DSS was $5 \%(\mathrm{w} / \mathrm{w})$ in tap water. Preventive administration was performed orally at $0.2 \mathrm{ml} /$ mouse/day for 7 days before DSS treatment. Cont., control; DSS, dextran sodium sulfate; (i)FP, (inactivated) Faecalibacterium prausnitzii; sup., supernatant.

Table II. DAI score assessment.

A, Fecal properties score

\begin{tabular}{lc}
\hline Fecal properties & Score \\
\hline Normal stools & 0 \\
Loose stools (tangible wet stools) & 1 \\
Diarrhea stools (collapsed wet stools) & 2 \\
Watery stools (liquid stools) & 3 \\
\hline
\end{tabular}

B, Hematochezia score

\begin{tabular}{lc}
\hline Hematochezia properties & Score \\
\hline Normal & 0 \\
Blood in the stool by visual & 1 \\
Blood in the anus & 2 \\
Bleeding from the anus & 3 \\
\hline
\end{tabular}

DAI scores were calculated as the sum of the fecal properties score and hematochezia score. DAI, disease activity index.

Fisher Scientific, Inc.) using SYBR ${ }^{\circledR}$ Premix Ex Taq ${ }^{\mathrm{TM}}$ II (Takara Bio, Inc.). qPCR conditions were: $95^{\circ} \mathrm{C}$ for $15 \mathrm{sec}$, then 40 cycles of $95^{\circ} \mathrm{C}$ for $15 \mathrm{sec}$ and $60^{\circ} \mathrm{C}$ for $31 \mathrm{sec}$. The expression of cytokine mRNA [tumor necrosis factor- $\alpha$ (TNF $\alpha$ ); interferon- $\gamma$ (IFN $\gamma)$; interleukin (IL)-10, IL-12 and IL-17A] was determined; $\beta$-actin was used as an internal control. The $2^{-\Delta \Delta C q}$ method was used for quantification (39). The sequences of the primer sets are presented in Table IV.

Statistical analysis. Data were analyzed using StadView version 5.0 (SAS Institute, Inc.). DAI scores over time and mice body weight changes over time were compared by one-way ANOVA followed by Fisher's protected least significant difference test. Student's t-test was used for comparing two groups. $\mathrm{P}<0.05$ was considered to indicate a statistically significant difference. Data are presented as the mean \pm standard deviation for all
Table III. Histological score assessment.

A, Epithelium score

Epithelial properties

Score

Normal morphology

0

Loss of goblet cells

Loss of goblet cells in large areas

1

Loss of crypts

Loss of crypts in large areas

2

3

4

$\mathrm{B}$, Infiltration score

Infiltration properties

Score

No infiltration

0

Infiltration around crypt bases

1

Infiltration reaching the muscularis mucosa

Extensive infiltration reaching the muscularis

mucosa and thickening of the mucosa with

abundant edema

Infiltration of the submucosa

4

The total histological score was calculated as the sum of the epithelium scores and infiltration scores.

Table IV. Primer list for RT-qPCR.

\begin{tabular}{ll}
\hline Cytokine & \multicolumn{1}{c}{ Primers } \\
\hline TNF $\alpha$ & F: 5'-ATCCGCGACGTGGAACTG-3' \\
& R: 5'-ACCGCCTGGAGTTCTGGAA-3' \\
IFN $\gamma$ & F: 5'-CCTGCGGCCTAGCTCTGA-3' \\
& R: 5'-CCATGAGGAAGAGCTGCAAAG-3' \\
IL-10 & F: 5-AGCAGCCTTGCAGAAAAGAGA-3' \\
& R: 5-AGTAAGAGCAGGCAGCATAGCA-3' \\
IL-12p35 & F: 5'-CACCCTTGCCCTCCTAAACC-3' \\
& R: 5'-CACCTGGCAGGTCCAGAGA-3' \\
IL-17A & F: 5'-TCATCTGTGTCTCTGATGCTGTTG-3' \\
& R: 5'-TCGCTGCTGCCTTCACTGT-3' \\
3-actin & F: 5'-TATCCACCTTCCAGCAGATGT-3' \\
& R: 5'-AGCTCAGTAACAGTCCGCCTA-3'
\end{tabular}

RT-qPCR, reverse transcription-quantitative polymerase chain reaction; IL, interleukin; IFN $\gamma$, interferon- $\gamma$; TNF $\alpha$, tumor necrosis factor- $\alpha$; F, forward; R, reverse.

experiments with the exception of RT-qPCR data, which are presented as the mean \pm standard error of the mean.

\section{Results}

Inflammation of DSS-induced colitis. The administered F. prausnitzii fluid $(0.2 \mathrm{ml})$ contained $\sim 1.5 \times 10^{8} \mathrm{CFU}$. All mice 
administered DSS developed diarrhea, bloody stools, weight loss and shortening of the colon, all symptoms of colitis (Figs. 1-4); however, these symptoms were alleviated in mice administered living $F$. prausnitzii prior to DSS administration (DSS + FP groups). Diarrhea and bloody stools were assessed using the DAI score; this value was significantly improved in the DSS + FP group compared with the DSS + Cont. group from day 6 onwards $(\mathrm{P}<0.01$; Fig. 1). Weight loss was also significantly alleviated in the DSS + FP group compared with the control (Fig. 2). DSS + FP group mice exhibited similar weight loss to the DSS + Cont. group until day 6; however, from day 7 , the relative weights of the mice were significantly increased in the DSS + FP group compared with the DSS + Cont. group. The weight of the DSS + Cont. group by day 8 was reduced to $86.2 \pm 4.2 \%$ of the starting weight, whereas that of the DSS + FP group was reduced to $89.7 \pm 5.2 \%$. The colon length of the DSS+FP group was significantly increased compared with the DSS + Cont. group (Fig. 3). The colon length of the DSS + Cont. group was reduced by $20.8 \pm 8.8 \%$, whereas that of the DSS + FP group was decreased by $15.2 \pm 6.8 \%$. It was observed that the administration of inactivated $F$. prausnitzii induced no protective effects against DSS-induced colitis in BALB/c mice. The DAI score, weight loss and colon length reduction were not ameliorated in mice administered inactivated bacterial materials (Fig. 4). In the absence of DSS treatment, when comparing the FP group and the Cont. group, no notable side effects of $F$. prausnitzii administration on weight change, DAI score, colon length, appearance or behavior were observed.

Histological analysis of the colon. The internal folds in the colons of mice administered DSS were reduced by inflammation (Fig. 5). The histological scores of the DSS + FP groups were significantly improved compared with the DSS + Cont. groups $(\mathrm{P}<0.05)$. Inflammation of the colon was alleviated in mice administered DSS with preventive $F$. prausnitzii compared with mice administered DSS alone.

Cytokine expression. The expression of TNF $\alpha$, IFN $\gamma$, IL-17A, IL-10 and IL-12 normalized to $\beta$-actin in the mucosa of the large intestine is presented in Fig. 6 . There were no significant differences in the expression of each cytokine upon comparing the DSS + FP group with the DSS + Cont. group; however, the expression levels of TNF $\alpha$, IFN $\gamma$ and IL-10 in the DSS + FP group were notably decreased compared with the DSS + Cont. group. The levels of IL-17A and IL-12 were markedly unaltered in the DSS + FP group compared with the DSS + Cont. group.

Therapeutic effect. For the therapeutic experiment, animals were first administered DSS, and then control or FP treatment. There were no significant differences between the DSS + Cont. and DSS + FP groups in terms of DAI score, weight change and colon length; however, the histological score of animals in the DSS + FP group was significantly improved (Fig. 7). Proinflammatory cytokine expression was measured by RT-qPCR. The expression of all cytokines analyzed during the experiment was markedly downregulated in the mucosa of the DSS + FP group compared with the DSS + Cont. group; however there were no significant differences (Fig. 8).

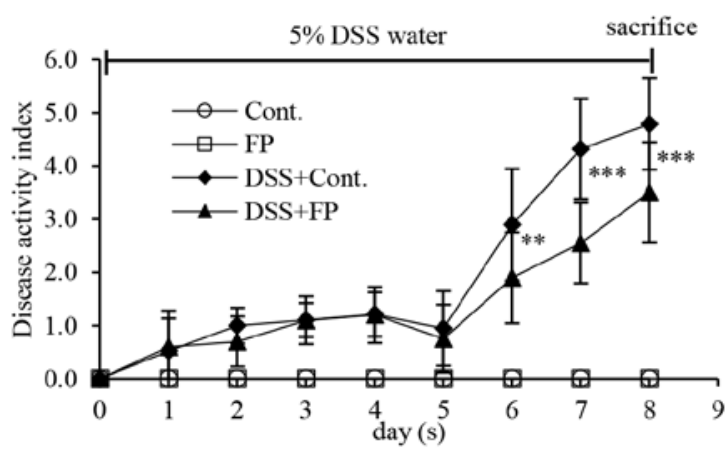

Figure 1. Effects of FP preventive treatment on the DAI score during DSS-induced colitis. The DAI score was measured daily, and was the sum of the fecal properties score and hematochezia score. Cont. group $(n=9)$; FP group $(n=8)$; DSS + Cont. group $(n=19)$; DSS + FP group $(n=20)$ Data are presented as the mean \pm standard deviation. ${ }^{* * *} \mathrm{P}<0.01,{ }^{* * *} \mathrm{P}<0.001$ vs. DSS + Cont. (Fisher's protected least significant difference test). Cont., control; DAI, disease activity index; DSS, dextran sodium sulfate; FP, Faecalibacterium prausnitzii.

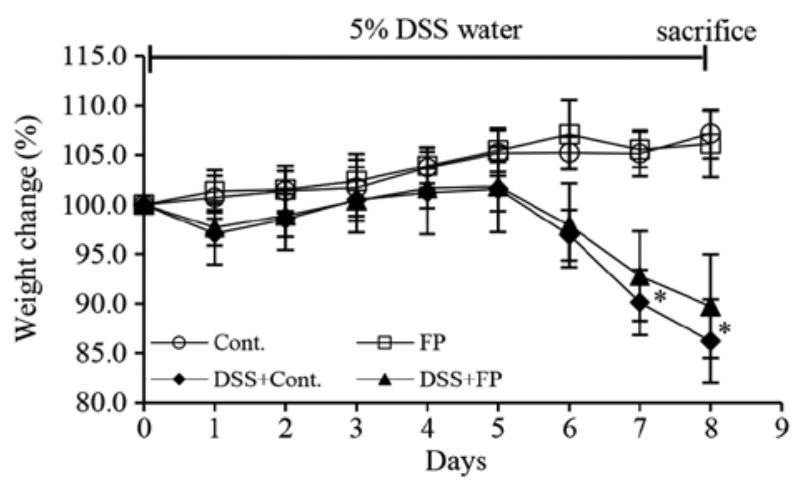

Figure 2. Effects of FP preventive treatment on body weight during DSS-induced colitis. Body weight was measured daily. Weight at day 0 was set as $100 \%$. Cont. group ( $n=9)$; FP group $(n=8)$; DSS + Cont. group $(n=19)$; DSS + FP group $(n=20)$. Data are presented as the mean \pm standard deviation. ${ }^{*} \mathrm{P}<0.05$ vs. DSS + Cont. (Fisher's protected least significant difference test). Cont., control; DSS, dextran sodium sulfate; FP, Faecalibacterium prausnitzii.

\section{Discussion}

Previous studies reported that $F$. prausnitzii is associated with IBD pathogenesis, and that certain patients with IBD were reported to exhibit smaller populations of $F$. prausnitzii compared with healthy counterparts (18-30). Furthermore, administration of $F$. prausnitzii was reported to improve chemically-induced colitis in murine models (18,31-33).

In the present study, live $F$. prausnitzii prevented DSS-induced colitis in BALB/c mice. The colon length was significantly increased in the live $F$. prausnitzii-administered group compared with the DSS + Cont. group, and a DAI assessment revealed that the damage mediated by DSS-induced colitis was reduced; however, administration of inactivated $F$. prausnitzii exhibited no effects on DSS-induced colitis. Therefore, it was suggested that live, but not inactivated $F$. prausnitzii protected the colon from DSS-induced colitis when administered prior to its onset. Therapeutic experiments were also performed; following the induction of colitis with DSS, live $F$. prausnitzii were administered during the 

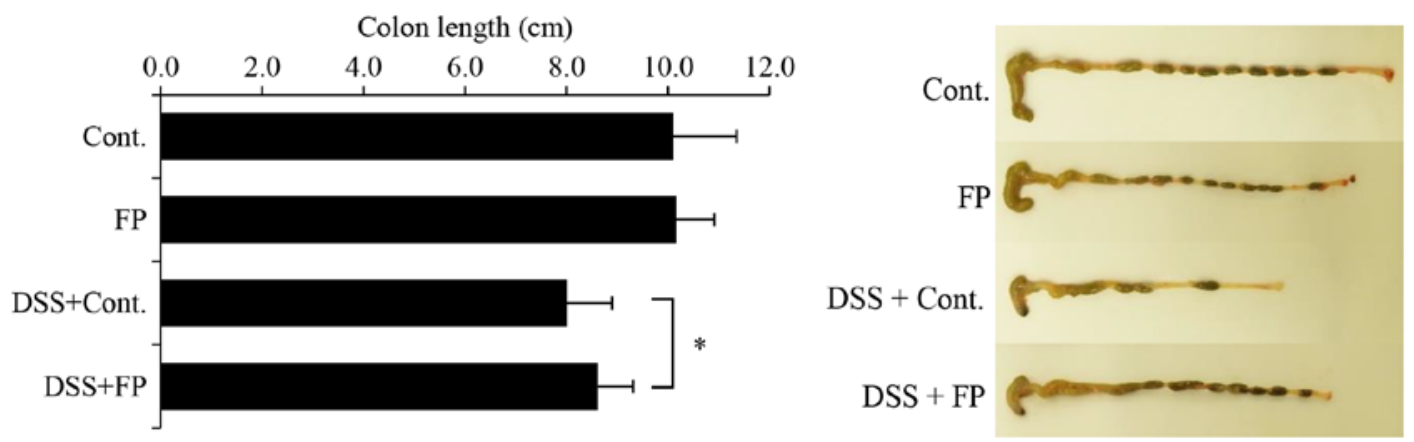

Figure 3. Effects of FP preventive treatment on colon length during DSS-induced colitis. Mean colon length and representative samples of dissected large intestines from mice in each group. Cont. group $(n=9)$; FP group $(n=8)$; DSS + Cont. group $(n=19)$; DSS + FP group $(n=20)$. Data are presented as the mean \pm standard deviation. ${ }^{*} \mathrm{P}<0.05$ vs. DSS + Cont. (Fisher's protected least significant difference test). Cont., control; DSS, dextran sodium sulfate; FP, Faecalibacterium prausnitzii.
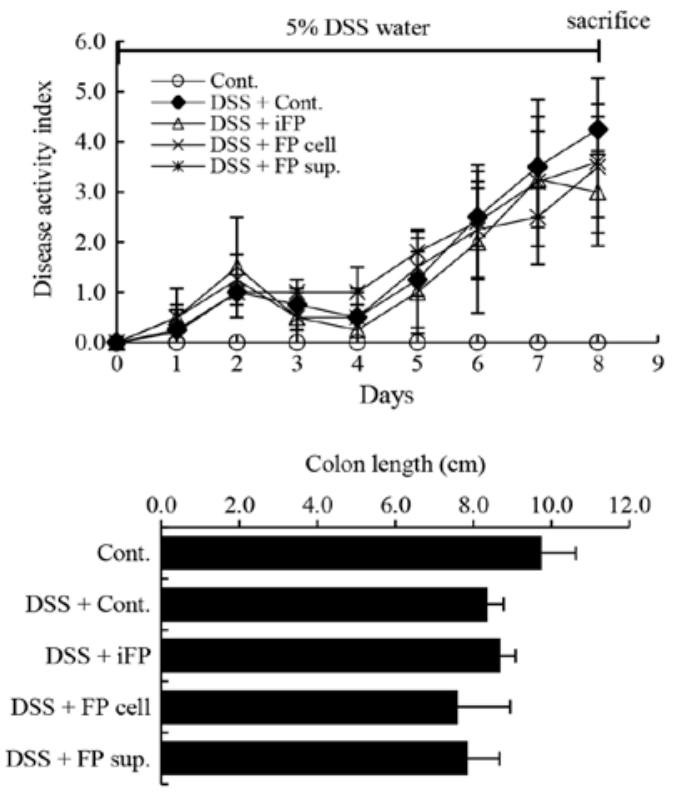
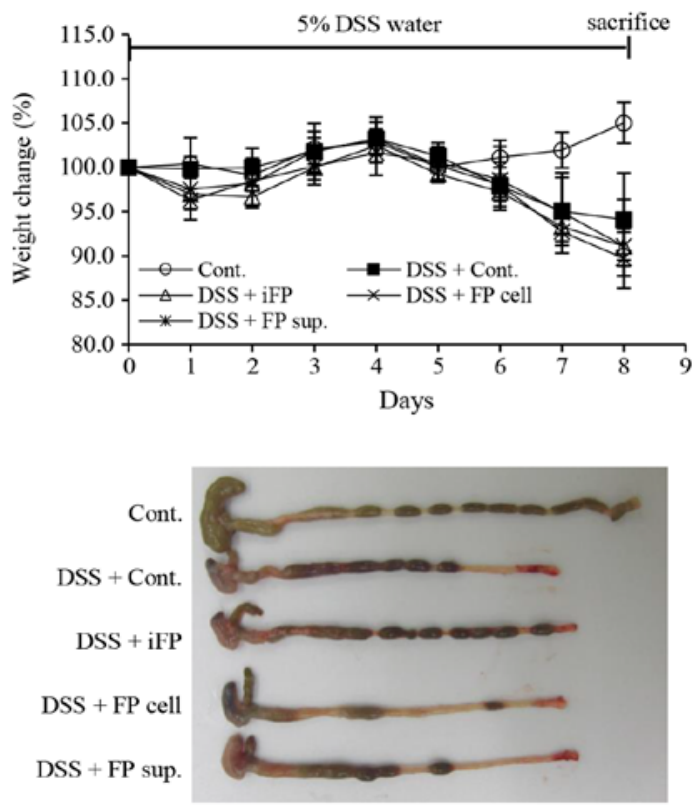

Figure 4. Inactivated FP does not protect the colon from DSS-induced colitis. DAI score and weight were measured daily. Mean colon length and representative samples of dissected large intestines from mice in each group are presented. There were no significant differences in any variable between the DSS groups. Cont. group $(n=5)$; DSS + Cont. group $(n=4)$; DSS + iFP group $(n=4)$; DSS + FP cell $(n=4)$; DSS + FP sup. $(n=5)$. Data are presented as the mean \pm standard deviation. Cont., control; DSS, dextran sodium sulfate; (i)FP, (inactivated) Faecalibacterium prausnitzii; sup., supernatant.
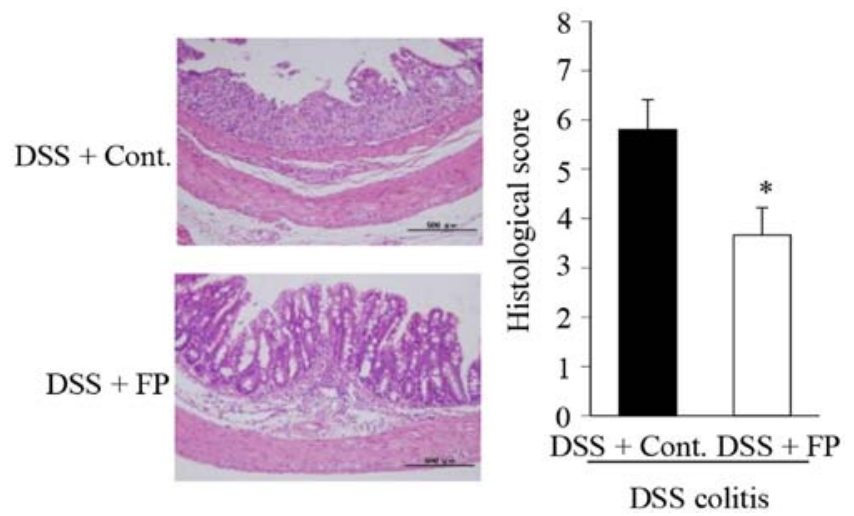

Figure 5. Histological analysis of the colon mucosa. H\&E-stained colonic mucosa from mice in the DSS + Cont. $(n=2)$ and DSS + FP groups $(n=2)$. The histological score was calculated as the sum of the epithelium score and infiltration score. Scale bar, $500 \mu \mathrm{m}$. Data are presented as the mean \pm standard deviation. ${ }^{*} \mathrm{P}<0.05$ vs. DSS + Cont. (Student's t-test). Cont., control; DSS, dextran sodium sulfate; FP, Faecalibacterium prausnitzii. recovery period. The DAI score and weight quickly recovered, but DSS-induced colitis was observed in the colon mucosa. The mice administered live $F$. prausnitzii exhibited improved recovery from DSS-induced colitis compared with the Cont. group, based on the histological analysis.

It was hypothesized that live $F$. prausnitzii regulated the expression of proinflammatory cytokines, thus protecting the colon from DSS-induced colitis. There were no significant differences in cytokine expression between the DSS + Cont. group and DSS + FP group in the preventive or therapeutic experiments; however, the expression of proinflammatory cytokines in the DSS + FP group was markedly decreased compared with in DSS + Cont. group. Anti-inflammatory cytokines are reported to improve chemically-induced colitis in mice (18). Rodent models have been frequently used to study colitis; colitis has been experimentally induced using various chemical agents, including DSS, 2,4,6-trinitrobenzene sulfonic acid (TNBS) and dinitrobenzene sulfonic 


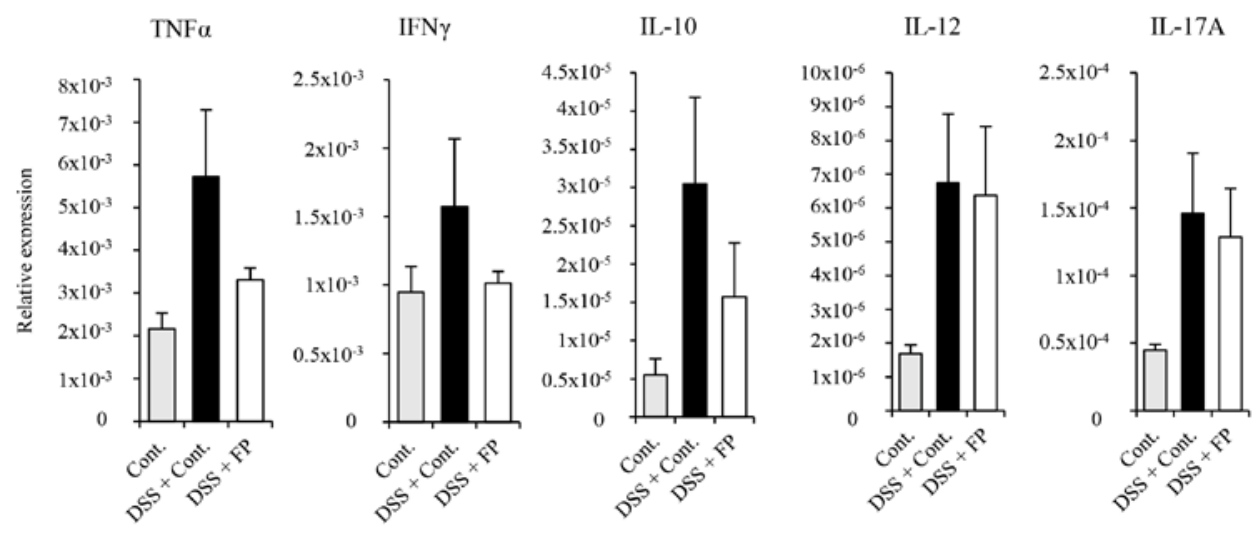

Figure 6. Expression of proinflammatory cytokines in the colon mucosa. Cytokine expression was measured via RT-qPCR. $\beta$-actin was used as an internal standard. RT-qPCR analysis was performed on 5 samples/group. Data are presented as the mean \pm standard error of the mean. Cont., control; DSS, dextran sodium sulfate; FP, Faecalibacterium prausnitzii; IL, interleukin; IFN $\gamma$, interferon- $\gamma$; RT-qPCR, reverse transcription-quantitative polymerase chain reaction; $\mathrm{TNF} \alpha$, tumor necrosis factor- $\alpha$.
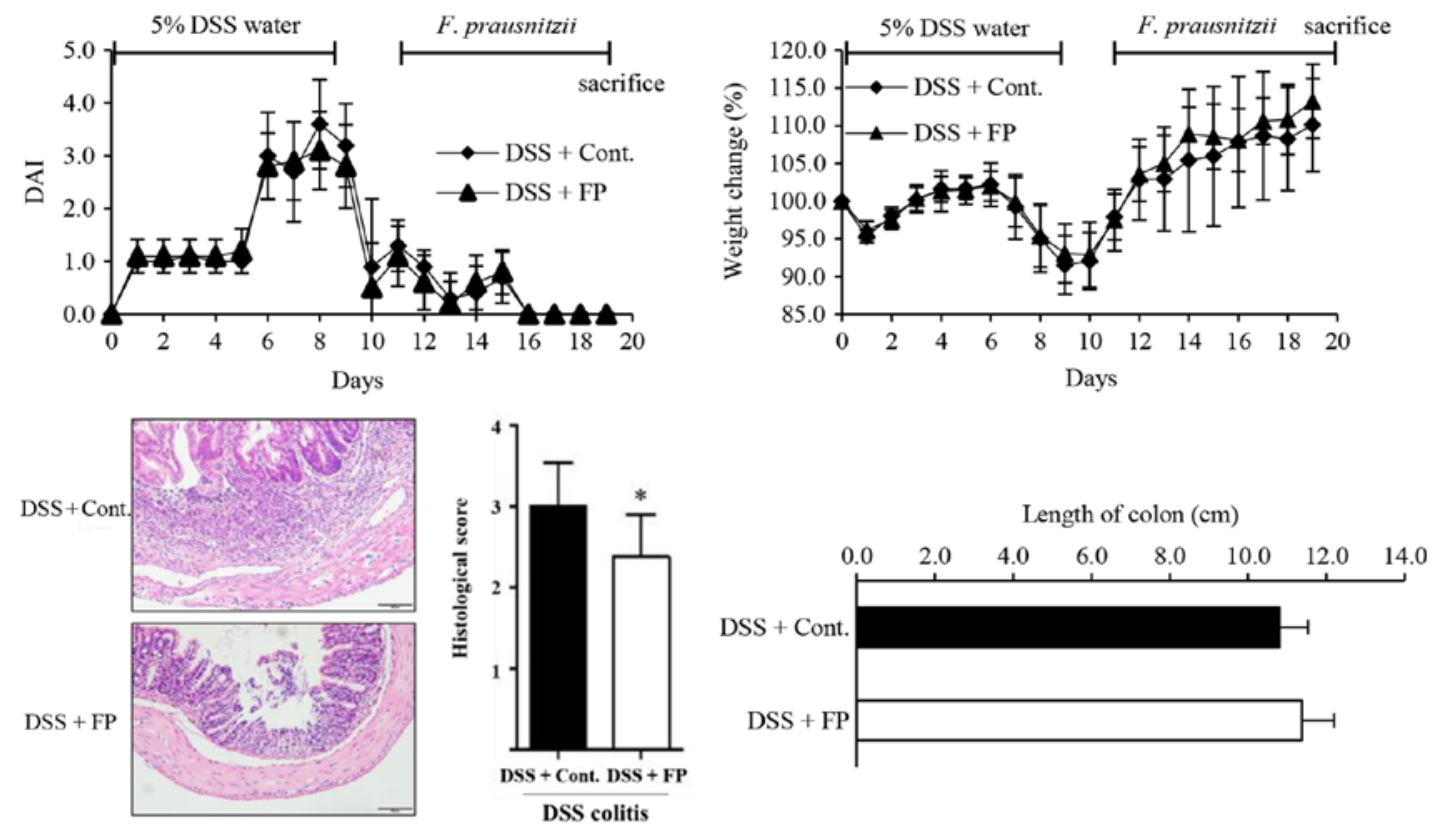

Figure 7. DAI score, weight change, colon length and histological score in the therapeutic experiment. Animals were administered DSS for 8 days, and then FP or control treatment for 7 days. DAI score and weight change were measured daily. DSS + Cont. group $(n=10)$; DSS + FP group ( $n=10)$. The colon length of each mouse was measured. Histological analyses were performed in 5 samples/group. Scale bar, $100 \mu \mathrm{m}$. Data are presented as the mean \pm standard deviation. ${ }^{*} \mathrm{P}<0.05$ vs. DSS + Cont. (Student's t-test). Cont., control; DAI, disease activity index; DSS, dextran sodium sulfate; FP, Faecalibacterium prausnitzii.
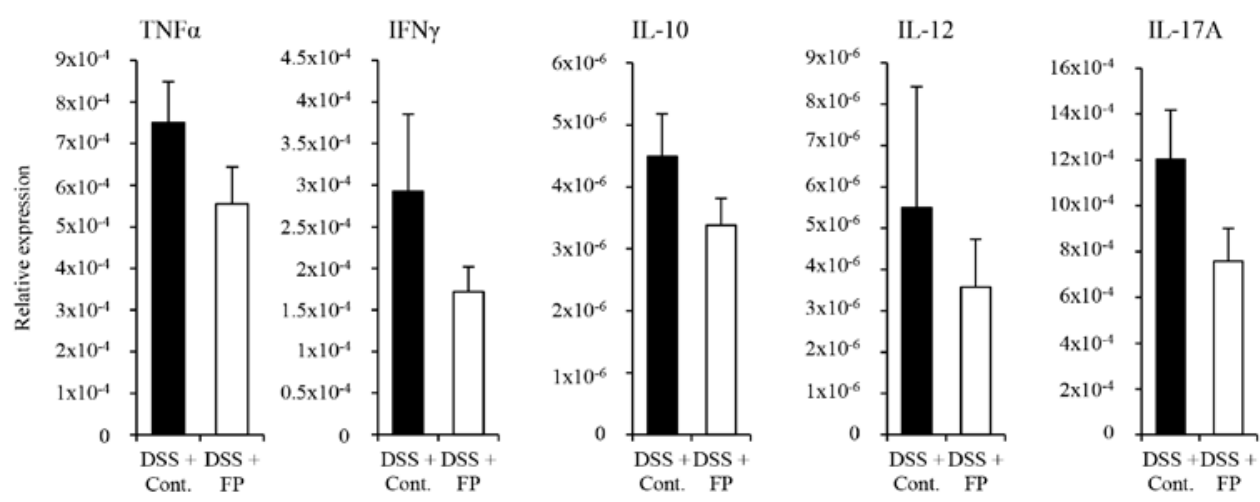

Figure 8. Proinflammatory cytokine expression in the colon mucosa during the therapeutic experiment. Animals were administered DSS for 8 days, and then FP or Control. Treatment for 7 days. Cytokine expression was measured via RT-qPCR. $\beta$-actin was used as an internal standard. RT-qPCR analysis was performed on 5 samples/group. Data are presented as the mean \pm standard error of the mean. Cont., control; DSS, dextran sodium sulfate; FP, Faecalibacterium prausnitzii; IL, interleukin; IFN $\gamma$, interferon- $\gamma$; RT-qPCR, reverse transcription-quantitative PCR; TNF $\alpha$, tumor necrosis factor- $\alpha$. 
acid (DNBS) (18,31-33). In the present study, $F$. prausnitzii protected the colon from chemically-induced colitis by regulating the expression of inflammatory cytokines, such as TNF- $\alpha$ and IL-12. Sokol et al (18), reported that the severity of TNBS-induced colitis in BALB/c mice was reduced by $F$. prausnitzii and its supernatants, with TNF- $\alpha$ and IL-12 secretion levels reduced compared with the vehicle and colitis control, and IL-10 secretion induced in the colons of mice treated with $F$. prausnitzii or its supernatant. Furthermore, Huang et al (31) reported that $F$. prausnitzii supernatant ameliorated DSS-induced colitis in C57BL/6J male mice via the inhibition of T-helper cell 17 differentiation and IL-17A secretion in plasma cells and colon mucosa. Rossi et al (32) reported that $F$. prausnitzii strain HTF-F and the extracellular polymeric matrix induced anti-inflammatory effects on the clinical parameters measured in the DSS model, with varying efficacy. These studies reported that the supernatant exhibited a preventive effect on chemical-induced colitis; however, there were no preventive effects following treatment with inactivated $F$. prausnitzii or its supernatant observed in the present study. Only live $F$. prausnitzii cells exhibited a preventive anti-inflammatory effect.

The mechanisms underlying the induction of colitis by DSS are unclear; DSS has been suggested to exert topical toxic effects on colonic epithelial cells, thereby disrupting mucosal barrier function $(34,40)$. The effects of DSS depend on its molecular weight; the greater the molecular weight of DSS, the more severe the symptoms $(34,40)$. Furthermore, TNBS and DNBS can bind covalently to the E-amino group of lysine and modify cell surface proteins on $\mathrm{T}$ lymphocytes, whereas reactive oxygen species generated via TNBS metabolism may induce colitis; these effects, combined with ethanol, then disrupt the mucosal barrier function (34). There are certain differences between DSS and TNBS; DSS affects the colonic mucosal barrier function, whereas TNBS affects immune function. Furthermore, the DSS used in this study had a lower molecular weight $(5,000 \mathrm{Da})$ than that used in other studies $(32,33)$. BALB/c mice were thus less sensitive to DSS-induced colitis than C57BL/6J mice (38). Therefore, DSS-induced colitis was considered to be milder compared with the other DSS-induced colitis studies.

It was hypothesized that administration of live $F$. prausnitzii in the present study improved mucosal barrier function, mucosal thickness, and mucin expression; consequently, the symptoms of DSS-induced colitis were reduced in BALB/c mice. In the present study, live $F$. prausnitzii appeared to exhibit anti-inflammatory effects; however, inactivated $F$. prausnitzii appeared to induce no effects on colitis. Therefore, it was hypothesized that $F$. prausnitzii may metabolize natural nutrients, polysaccharides or other molecules into anti-inflammatory substances in the process of growth or during the colonization of the intestinal mucosa, which then interact with other gut microbes. Further studies into the mucosal barrier system and the events occurring in the intestine upon the administration of $F$. prausnitzii are required.

\section{Acknowledgements}

Not applicable.

\section{Funding}

The present study was supported by a Grant-in-Aid for Scientific Research (KAKENHI) to YN (grant no. 16H05289) from the Japan Society for the Promotion of Science, and R\&D Matching Funds on the Field for Knowledge Integration and Innovation by National Agriculture and Food Research Organization. This research was also supported by the Matching Planner Program of the Japan Science and Technology Agency (grant no. MP28116808763).

\section{Availability of data and materials}

The datasets used and/or analyzed during the current study are available from the corresponding author on reasonable request.

\section{Authors' contributions}

YK and TS made substantial contributions to the conception and design of the study and experiments. YK, MS, MO, MM, KM, TU, TT and TS performed the experiments. YK, YN, YI and TS analyzed the data. YK, YN, YI and TS drafted the manuscript.

\section{Ethics approval and consent to participate}

All the experimental procedures using mice were conducted in accordance with the National Institutes of Health guidelines for the use of experimental animals. Experiments were performed according to the guidelines for the care and use of laboratory animals approved by Nichinichi Pharmaceutical Co. Ltd. All experimental programs were approved by the Animal Care Committee of Kyoto Prefectural University of Medicine (Kyoto, Japan).

\section{Patient consent for publication}

Not applicable.

\section{Competing interests}

The authors declare that they have no competing interests.

\section{References}

1. CatoEP,Salmon CW and Moore WEC: Fusobacterium prausnitzii (Hauduroy et al.) moore and holdeman: emended description and designation of neotype strain. Int J Syst Bacteriol 24: 225-229, 1974.

2. Duncan SH, Hold GL, Harmsen HJM, Stewart CS and Flint HJ: Growth requirements and fermentation products of Fusobacterium prausnitzii, and a proposal to reclassify it as Faecalibacterium prausnitzii gen. nov., comb. nov. Int J Syst Evol Microbiol 52: 2141-2146, 2002.

3. Lopez-Siles M, Khan TM, Duncan SH, Harmsen HJM, Garcia-Gil LJ and Flint HJ: Cultured representatives of two major phylogroups of human colonic Faecalibacterium prausnitzii can utilize pectin, uronic acids, and host-derived substrates for growth. Appl Environ Microbiol 78: 420-428, 2012.

4. Lopez-Siles M, Martinez-Medina M, Busquets D, Sabat-Mir M Duncan SH, Flint HJ, Aldeguer X and Garcia-Gil LJ: Mucosa-associated Faecalibacterium prausnitzii and Escherichia coli co-abundance can distinguish irritable bowel syndrome and inflammatory bowel disease phenotypes. Int $\mathbf{J}$ Med Microbiol 304: 464-475, 2014. 
5. Nava GM and Stappenbeck TS: Diversity of the autochthonous colonic microbiota. Gut Microbes 2: 99-104, 2011.

6. Baumgart DC and Sandborn WJ: Inflammatory bowel disease: Clinical aspects and established and evolving therapies. Lancet 369: 1641-1657, 2007

7. Arumugam M, Raes J, Pelletier E, Le Paslier D, Yamada T, Mende DR, Fernandes GR, Tap J, Bruls T, Batto JM, et al: Enterotypes of the human gut microbiome. Nature 473: 174-780, 2011.

8. Hold GL, Schwiertz A, Aminov RI, Blaut M and Flint HJ: Oligonucleotide probes that detect quantitatively significant groups of butyrate-producing bacteria in human feces. Appl Environ Microbiol 69: 4320-4324, 2003.

9. Schwiertz A, Jacobi M, Frick JS, Richter M, Rusch K and Köhler H: Microbiota in pediatric inflammatory bowel disease. J Pediatr 157: 240-244.e1, 2010.

10. Suau A, Rochet V, Sghir A, Gramet G, Brewaeys S, Sutren M, Rigottier-Gois L and Doré J: Fusobacterium prausnitzii and related species represent a dominant group within the human fecal flora. Syst Appl Microbiol 24: 139-145, 2001.

11. Walker AW, Ince J, Duncan SH, Webster LM, Holtrop G, Ze X, Brown D, Stares MD, Scott P, Bergerat A, et al: Dominant and diet-responsive groups of bacteria within the human colonic microbiota. ISME J 5: 220-230, 2011

12. Swidsinski A, Loening-Baucke V, Lochs H and Hale LP: Spatial organization of bacterial flora in normal and inflamed intestine: A fluorescence in situ hybridization study in mice. World J Gastroenterol 11: 1131-1140, 2005.

13. Hopkins MJ, Macfarlane GT, Furrie E, Fite A and Macfarlane S: Characterisation of intestinal bacteria in infant stools using real-time PCR and northern hybridisation analyses. FEMS Microbiol Ecol 54: 77-85, 2005.

14. Balamurugan R, Janardhan HP, George S, Chittaranjan SP and Ramakrishna BS: Bacterial succession in the colon during childhood and adolescence: Molecular studies in a southern Indian village. Am J Clin Nutr 88: 1643-1647, 2008.

15. Wang M, Ahrné S, Antonsson M and Molin G: T-RFLP combined with principal component analysis and $16 \mathrm{~S}$ rRNA gene sequencing: An effective strategy for comparison of fecal microbiota in infants of different ages. J Microbiol Methods 59: 53-69, 2004

16. Fallani M, Rigottier-Gois L, Aguilera M, Bridonneau C, Collignon A, Edwards CA, Corthier G and Doré J: Clostridium difficile and Clostridium perfringens species detected in infant faecal microbiota using 16S rRNA targeted probes. J Microbiol Methods 67: 150-161, 2006.

17. Lopez-Siles M, Duncan SH, Garcia-Gil LJ and Martinez-Medina M: Faecalibacterium prausnitzii: From microbiology to diagnostics and prognostics. ISME J 11, 841-852, 2017

18. Sokol H, Pigneur B, Watterlot L, Lakhdari O Bermúdez-Humarán LG, Gratadoux JJ,Blugeon S, Bridonneau C, Furet JP, Corthier G, et al: Faecalibacterium prausnitzii is an anti-inflammatory commensal bacterium identified by gut microbiota analysis of Crohn disease patients. Proc Natl Acad Sci USA 105: 16731-16736, 2008.

19. Miquel S, Martín R, Rossi O, Bermúdez-Humarán LG, Chatel JM, Sokol H, Thomas M, Wells JM and Langella P: Faecalibacterium prausnitzii and human intestinal health. Curr Opin Microbiol 16: 255-261, 2013.

20. Frank DN, St Amand AL, Feldman RA, Boedeker EC, Harpaz N and Pace NR: Molecular-phylogenetic characterization of microbial community imbalances in human inflammatory bowel diseases. Proc Natl Acad Sci USA 104: 13780-13785, 2007.

21. Martinez-Medina M, Aldeguer X, Gonzalez-Huix F, Acero D and Garcia-Gil LJ: Abnormal microbiota composition in the ileocolonic mucosa of Crohn's disease patients as revealed by polymerase chain reaction-denaturing gradient gel electrophoresis. Inflamm Bowel Dis 12: 1136-1145, 2006.

22. Sokol H, Seksik P, Furet JP, Firmesse O, Nion-Larmurier I, Beaugerie L, Cosnes J, Corthier G, Marteau P and Doré J: Low counts of Faecalibacterium prausnitzii in colitis microbiota. Inflamm Bowel Dis 15: 1183-1189, 2009.

23. Swidsinski A, Loening-Baucke V, Vaneechoutte $M$ and Doerffel Y: Active Crohn's disease and ulcerative colitis can be specifically diagnosed and monitored based on the biostructure of the fecal flora. Inflamm Bowel Dis 14: 147-161, 2008.
24. Willing B, Halfvarson J, Dicksved J, Rosenquist M, Järnerot G, Engstrand L, Tysk C and Jansson JK: Twin studies reveal specific imbalances in the mucosa-associated microbiota of patients with ileal Crohn's disease. Inflamm Bowel Dis 15: 653-660, 2009.

25. Furet JP, Kong LC, Tap J, Poitou C, Basdevant A, Bouillot JL, Mariat D, Corthier G, Doré J, Henegar C, et al: Differential Adaptation of Human Gut Microbiota to bariatric surgery-induced weight loss: Links with metabolic and low-grade inflammation markers. Diabetes 59: 3049-3057, 2010.

26. Hansen R, Russell RK, Reiff C, Louis P, McIntosh F, Berry SH, Mukhopadhya I,Bisset WM, Barclay AR,Bishop J, et al: Microbiota of de-novo pediatric IBD: Increased Faecalibacterium prausnitzii and reduced bacterial diversity in Crohn's but not in ulcerative colitis. Am J Gastroenterol 107: 1913-1922, 2012.

27. Jia W, Whitehead RN, Griffiths L, Dawson C, Waring RH, Ramsden DB, Hunter JO and Cole JA: Is the abundance of Faecalibacterium prausnitzii relevant to Crohn's disease? FEMS Microbiol Lett 310: 138-144, 2010.

28. Kabeerdoss J, Sankaran V, Pugazhendhi S and Ramakrishna BS: Clostridium leptum group bacteria abundance and diversity in the fecal microbiota of patients with inflammatory bowel disease: A case-control study in India. BMC Gastroenterol 13: 20, 2013.

29. Machiels K, Joossens M, Sabino J, De Preter V, Arijs I, Eeckhaut V, Ballet V, Claes K, Van Immerseel F, Verbeke K, et al: A decrease of the butyrate-producing species Roseburia hominis and Faecalibacterium prausnitzii defines dysbiosis in patients with ulcerative colitis. Gut 63: 1275-1283, 2014.

30. Vermeiren J, van den Abbeele P, Laukens D, Vigsnæs LK, de Vos M, Boon N and van de Wiele T: Decreased colonization of fecal Clostridium coccoides/Eubacterium rectale species from ulcerative colitis patients in an in vitro dynamic gut model with mucin environment. FEMS Microbiol Ecol 79: 685-696, 2012.

31. Huang XL, Zhang X, Fei XY, Chen ZG, Hao YP, Zhang S, Zhang MM, Yu YQ and Yu CG: Faecalibacterium prausnitzii supernatant ameliorates dextran sulfate sodium induced colitis by regulating Th17 cell differentiation. World J Gastroenterol 22: 5201-5210, 2016.

32. Rossi O, Khan MT, Schwarzer M, Hudcovic T, Srutkova D, Duncan SH, Stolte EH, Kozakova H, Flint HJ, Samsom JN, et al: Faecalibacterium prausnitzii strain HTF-F and its extracellular polymeric matrix attenuate clinical parameters in DSS-induced colitis. PLoS One 10: e0123013, 2015.

33. Breyner NM, Michon C, de Sousa CS, Vilas Boas PB, Chain F, Azevedo VA, Langella P and Chatel JM: Microbial anti-inflammatory molecule (MAM) from Faecalibacterium prausnitzii shows a protective effect on DNBS and DSS-induced colitis model in mice through inhibition of NF- $\mathrm{B}$ pathway. Front Microbiol 8: 114, 2017.

34. Tran CD, Katsikeros R and Abimosleh SM: Current and novel treatments for ulcerative colitis, Ulcerative Colitis from Genetics to Complications, Prof. Mustafa Shennak (ed) ISBN: 978-953-307-853-3, InTech, 2012 http://www.intechopen. com/books/ulcerative-colitis-from-genetics-to-complications/currentand-novel-treatments-for-ulcerative-colitis.

35. Hungate RE: Chapter IV a roll tube method for cultivation of strict anaerobes. Methods Microbiol 3: 117-132, 1969.

36. National Research Council (US) Committee for the Update of the Guide for the Care and Use of Laboratory Animals. Guide for the Care and Use of Laboratory Animals. 8th edition. National Academies Press (US), Washington, DC, 2011. https://www.ncbi. nlm.nih.gov/books/NBK54050.

37. Hausmann M, Obermeier F, Paper DH, Balan K, Dunger N, Menzel K, Falk W, Schoelmerich J, Herfarth H and Rogler G: In vivo treatment with the herbal phenylethanoid acteoside ameliorates intestinal inflammation in dextran sulphate sodium-induced colitis. Clin Exp Immunol 148: 373-381, 2007.

38. Takagi T, Naito Y, Uchiyama K, Suzuki T, Hirata I, Mizushima K, Tsuboi H,Hayashi N, Handa O, Ishikawa T, et al: Carbon monoxide liberated from carbon monoxide-releasing molecule exerts an anti-inflammatory effect on dextran sulfate sodium-induced colitis in mice. Dig Dis Sci 56: 1663-1671, 2011

39. Livak KJ and Schmittgen TD: Analysis of relative gene expression data using real-time quantitative PCR and the 2(-Delta Delta C(T)) method. Methods 25: 402-408, 2001.

40. Chassaing B, Aitken JD, Malleshappa M and Vijay-Kumar M: Dextran sulfate sodium (DSS)-induced colitis in mice. Curr Protoc Immunol 104: Unit 15.25, 2014. 